\title{
Teleportation-Induced Correlated Quantum Channels
}

\author{
F. Caruso ${ }^{1,2,3}$, V. Giovannetti ${ }^{3}$, and G. M. Palma ${ }^{4}$ \\ ${ }^{1}$ Institute for Mathematical Sciences, 53 Prince's Gate, Imperial College, London, SW7 2PG, UK \\ ${ }^{2}$ QOLS, The Blackett Laboratory, Imperial College, London, Prince Consort Road, SW7 2BW, UK \\ ${ }^{3}$ NEST-CNR-INFM \& Scuola Normale Superiore, Piazza dei Cavalieri 7, I-56126, Pisa, Italy and \\ ${ }^{4}$ NEST-CNR - INFM \& Dipartimento di Scienze Fisiche ed Astronomiche, \\ Universita' degli studi di Palermo, via Archirafi 36, Palermo, I-90123, Italy
}

(Dated: October 24, 2018)

\begin{abstract}
Quantum teleportation of a $n$-qubit state performed using as entangled resource a general bipartite state of $2 n$ qubits instead of $n$ Bell states is equivalent to a correlated Pauli channel. This provides a new characterization of such channels in terms of many-body correlation functions of the teleporting media. Our model is then generalized to the Continuous Variable case. We show that this new representation provides a relatively simple method for determining whether a correlated quantum channel is able to reliably convey quantum messages by studying the entanglement properties of the teleportation mediating system.
\end{abstract}

PACS numbers: 03.67.-a, 03.67.Hk

Starting from the seminal works of Ref. [1] an increasing attention has been devoted to the study of the so called memory or, more precisely, Correlated Quantum (CQ) communication channels both at theoretical [2, 3, 4] and experimental level [5]. Differently from the standard memoryless configurations, a CQ channel is characterized by the presence of correlated noise sources which operate jointly on otherwise independently transmitted messages (channel uses in jargon). Recently these communication lines were put in correspondence with the physics of many-body quantum systems [6, 7, 8], by representing their noise effects in terms of unitary couplings connecting the transmitted signals with a many-body correlated quantum environment. In particular, Ref. [7] pointed out the possibility of relating the capacity of $\mathrm{CQ}$ channels with environmental critical phenomena. In this paper we introduce an alternative approach to the representation of CQ channels based on the teleportation representation of the (memoryless) depolarizing channels given by Bowen and Bose [9] and on its continuous variable generalization [10]. This provides an alternative characterization of the noise correlations of the channel in terms of many-body correlation functions of the medium that is employed in the teleportation with respect to previously analyzed models [6, 7, 8]. Furthermore the representation introduced here provides a relatively simple method for determining how reliable a CQ channel is to transmit quantum information by analyzing the entanglement properties of the physical system which mediates the teleportation. We start presenting the model for finite dimensional system postponing the continuous variable generalization to the second part of the paper.

Qubit CQ channels:- Consider $n$ independent quantum teleportation events [11] in which the entangled resource initially shared by the communicating parties (Alice and Bob) is not the correct Bell state $\left|\Psi_{0}\right\rangle^{\otimes n} \equiv$ $[(|00\rangle+|11\rangle) / \sqrt{2}]^{\otimes n}$ but a bipartite (not necessarily pure) $2 n$-qubit state $\chi^{(n)}$ - the bipartition being such that Alice

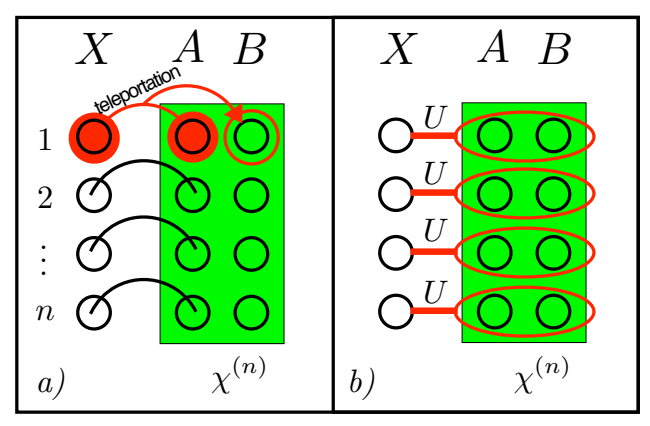

FIG. 1: Part a): Alice transfers messages encoded into the input qubits $X$ into the $B$ part of the multi-particle medium $\chi^{(n)}$ (green box) by teleportation (i.e. performing Bell measurements on $X A$ and communicating to Bob the local operations he has to perform on $B$ ). Part $b$ ): both the input and output states are written in $X$ while $\chi^{(n)}$ plays the role of an external (possibly) correlated environment - see Eq. (8). In both scenarios the resulting mapping is given by Eq. (11).

has access on the first $n$ qubits $A$, while Bob on the second $n$ qubits $B$. For $n=1$ it has been shown [9] that the transferring of a generic qubit state $\rho$ from Alice to Bob can be seen as the result of the depolarizing (or Pauli) channel $\Lambda^{(1)}(\rho)=\sum_{k=0}^{3} p_{k} \sigma_{k} \rho \sigma_{k}$, where $\sigma_{k}$ is the $k$-th Pauli operator, and where $p_{k}$ are the probabilities $p_{k}:=$ $\operatorname{Tr}\left[E_{k} \chi^{(n)}\right]$ with $E_{k}:=\left|\Psi_{k}\right\rangle\left\langle\Psi_{k}\right|=\left(\sigma_{k} \otimes \mathbb{1}\right) E_{0}\left(\sigma_{k} \otimes \mathbb{1}\right)$ being the projectors on the Bell basis $\left|\Psi_{k}\right\rangle$ - see Fig. 1 a). By the linearity this can be generalized to the $n>1$ case yielding

$$
\Lambda^{(n)}(\rho)=\sum_{\mathbf{k}} p_{\mathbf{k}}^{(n)} \sigma_{\mathbf{k}} \rho \sigma_{\mathbf{k}},
$$

where now $\rho$ is a generic input state of $n$ qubits, and for $\mathbf{k}:=\left(k_{1}, k_{2}, \cdots, k_{n}\right)$ one has $\sigma_{\mathbf{k}}:=\sigma_{k_{1}} \otimes \cdots \otimes \sigma_{k_{n}}$ and

$$
p_{\mathbf{k}}^{(n)}:=\operatorname{Tr}\left[E_{\mathbf{k}} \chi^{(n)}\right]
$$


with $E_{\mathbf{k}}:=E_{k_{1}} \otimes \cdots \otimes E_{k_{n}}$. Equation (1) describes a $n$-qubit correlated Pauli channel in which the rotations $\sigma_{\mathbf{k}}$ are randomly applied to the system according to the joint probability distribution $p_{\mathbf{k}}$ of finding $\chi^{(n)}$ into the state $\left|\Psi_{\mathbf{k}}\right\rangle:=\left|\Psi_{k_{1}}\right\rangle \otimes \cdots \otimes\left|\Psi_{k_{n}}\right\rangle$. Perfect teleportation is obtained when $\chi^{(n)}$ coincides with $E_{0}^{\otimes n}$ : in this case $p_{\mathbf{k}}^{(n)}=\delta_{k_{1} 0} \cdots \delta_{k_{n} 0}$ and $\Lambda^{(n)}$ becomes the identity channel. Vice-versa total depolarization is obtained, for instance, by using the complete mixed state $\chi^{(n)}=\mathbb{1} / 2^{2 n}$ (here $p_{\mathbf{k}}^{(n)}=1 / 2^{2 n}$ and $\Lambda^{(n)}(\rho)=\mathbb{1} / 2^{n}$ for all $\rho$ ). It is finally worth pointing out that the qubit $\mathrm{CQ}$ channels analyzed in Ref. 7] are included as the special case of our teleportation model, where all the $n$ shared couples of $\chi^{(n)}$ have vanishing projections on the vectors $\left|\Psi_{1,2}\right\rangle$. Pauli channels form a convex set which is closed under the super-operator composition. Specifically concatenating two Pauli maps $\Lambda_{1}^{(n)}$ and $\Lambda_{2}^{(n)}$ the result is still a Pauli channel $\Lambda_{3}^{(n)}=\Lambda_{2}^{(n)} \circ \Lambda_{1}^{(n)}=\Lambda_{1}^{(n)} \circ \Lambda_{2}^{(n)}$ whose probabilities (2) are obtained by a double-stochastic convolution of the previous ones (the order of the composition being irrelevant). Vice-versa any Pauli channel $\Lambda_{3}^{(n)}$ obtained by transforming the probabilities of $\Lambda_{1}^{(n)}$ via a double-stochastic transformation can be expressed as $\Lambda_{2}^{(n)} \circ \Lambda_{1}^{(n)}\left(\right.$ or $\left.\Lambda_{1}^{(n)} \circ \Lambda_{2}^{(n)}\right)$ for some proper choice of $\Lambda_{2}^{(n)}$. Expressed in terms of the teleportation representation (11) these properties translate into the following identities $\chi_{3}^{(n)}=\Lambda_{1}^{(n)}\left(\chi_{2}^{(n)}\right)$ or $\chi_{3}^{(n)}=\Lambda_{2}^{(n)}\left(\chi_{1}^{(n)}\right)$ - both choices yield the same probabilities (2).

CJ isomorphism:- The teleportation representation provides a many to one correspondence among $2 n$-qubit states $\chi^{(n)}$ and $n$-qubit correlated Pauli channels. Indeed, since the off-diagonal terms of the $\chi^{(n)} \mathrm{s}$ in the Bell basis do not influence the resulting $p_{\mathbf{k}}^{(n)}$, we can associate more than one teleportation mediator for each $\Lambda^{(n)}$. The simplest choice is of course to use the Bell-diagonal state $\chi_{B D}^{(n)}:=\sum_{\mathbf{k}} p_{\mathbf{k}}^{(n)} E_{\mathbf{k}}$. Interestingly enough this also coincides with the Choi-Jamiolkowski (CJ) state of $\Lambda^{(n)}$ [12]. We remind that the latter is a bipartite state associated with $\Lambda^{(n)}$ obtained by applying the channel on half of a maximally entangled state $|\Psi\rangle$ of the system and of an extra ancillary system, i.e. $\left(\Lambda^{(n)} \otimes I\right)(|\Psi\rangle\langle\Psi|)$ (here $I$ is the identity super-operator on the ancilla). Its entropy and fidelity with $|\Psi\rangle$ coincide respectively with channel entropy [13] and the entanglement fidelity [14] of $\Lambda^{(n)}$ that were used in Ref. [8] to characterize the correlations of CQ channels. For the CQ channel of Eq. (1) we can take $|\Psi\rangle:=\left|\Psi_{0}\right\rangle^{\otimes n}$ yielding the following C.J state,

$$
\left(\Lambda^{(n)} \otimes I\right)\left(E_{0}^{\otimes n}\right)=\sum_{\mathbf{k}} p_{\mathbf{k}}^{(n)} E_{\mathbf{k}}=\chi_{B D}^{(n)},
$$

as anticipated. In Ref. [15] it is shown that a (memoryless) channel with a Bell-diagonal CJ state possesses an useful symmetry (i.e., covariance property wrt Pauli rotations) in such a way that having one use of that channel is both mathematically and physically equivalent to having one copy of its CJ state shared among the sender and the receiver of the message. Therefore, by following the results of Ref. [15] and similar arguments as in Ref. 7], the quantum capacity [16] of any family $\mathcal{L}:=\left\{\Lambda^{(n)}: n \in \mathbb{N}\right\}$ of CQ channels (11) can be shown to obey the following inequality

$$
Q(\mathcal{L}) \leq \lim _{n \rightarrow \infty} \frac{D_{1}\left(\chi_{B D}^{(n)}\right)}{n},
$$

where, for each $n, \chi_{B D}^{(n)}$ is the CJ state (3) of $\Lambda^{(n)}$, and $D_{1}$ is the number of e-bits we can extract from an asymptotically large number of copies of $\chi_{B D}^{(n)}$ through one-way LOCC operations. As a matter of fact, the inequality (4) holds also if we replace $\chi_{B D}^{(n)}$ with a generic $\chi^{(n)}$ that provides a teleportation representation (2) for the maps $\Lambda^{(n)} \in \mathcal{L}$ (this trivially follows by fact that the direct teleportation through $\chi^{(n)}$ of e-bits is a special example of one-way distillation). In particular, this implies that $Q=0$ for $\mathcal{L}$ admitting separable teleportation media $\chi^{(n)}$. For forgetful channels [3] (i.e. for $\mathcal{L}$ having exponentially decreasing correlations, e.g. Markov or memoryless channels) the upper bound in Eq. (4) is tight. Thus, for these maps, by exploiting the hashing bound [15, 17], we can write

$$
Q(\mathcal{L})=\lim _{n \rightarrow \infty} \frac{D_{1}\left(\chi_{B D}^{(n)}\right)}{n} \geq 1-\lim _{n \rightarrow \infty} \frac{S\left(\chi_{B D}^{(n)}\right)}{n},
$$

with $S\left(\chi_{B D}^{(n)}\right)=-\sum_{\mathbf{k}} p_{\mathbf{k}}^{(n)} \log _{2} p_{\mathbf{k}}^{(n)}$ being the channel entropy [13] of $\Lambda^{(n)}$. This expression can be further simplified when $\chi_{B D}^{(n)}$ is a maximally correlated state [18]. In this case the inequality (5) saturates and reduces to Eq. (16) of Ref. 7] (see also Ref. [19]). It is finally worth pointing out that for all (non necessarily forgetful) CQ channels a upper bound [3] can be obtained in terms of the (regularized) coherent information $J\left(\rho, \Lambda^{(n)}\right):=S\left(\Lambda^{(n)}(\rho)\right)-S\left(\left(\Lambda^{(n)} \otimes I\right)\left(\left|\Psi_{\rho}\right\rangle\left\langle\Psi_{\rho}\right|\right)\right)$ of the channel, i.e.

$$
Q(\mathcal{L}) \leq \lim _{n \rightarrow \infty} \max _{\rho} J\left(\rho, \Lambda^{(n)}\right) / n
$$

(in this expression $\left|\Psi_{\rho}\right\rangle$ is a purification of $\rho$ ).

Equivalence with other representations:- We now show how one can recast the channel (1) in terms of an interaction with a many-body environment as in Refs. [6, 7, 8]. To do so we introduce the unitary operator

$$
U^{(n)}:=\sum_{\mathbf{k}} \sigma_{\mathbf{k}} \otimes E_{\mathbf{k}}=\otimes_{j=1}^{n} U_{j},
$$

with $U_{j}=\sum_{k_{j}} \sigma_{k_{j}} \otimes E_{k_{j}}$ the transformation which rotates by $\sigma_{k_{j}}$ the $j$-th qubit of the input ( $X$ system of part b) of Fig. 1) while projecting on the $k_{j}$-th Bell state the corresponding couples of sites of teleporting medium 
$\chi^{(n)}\left(A B\right.$ system of the figure). $U^{(n)}$ is thus a product of identical transformations which act separately with each input qubit. The equivalence then simply follows by the identity

$$
\Lambda^{(n)}(\rho)=\operatorname{Tr}_{A B}\left[U^{(n)}\left(\rho \otimes \chi^{(n)}\right)\left[U^{(n)}\right]^{\dagger}\right],
$$

where the partial trace is performed over the teleporting medium. In this representation $\chi^{(n)}$ plays the role of the many-body environment of the channel. This observation allows us to take advantage of the results of Ref. 7] to simplify the analysis of the quantum capacity of our teleportation representation. Specifically, Plenio and Virmani showed that the quantum capacity of the channel $\Lambda^{(n)}$ can be expressed as in the rhs term of Eq. (5) if the many-body environmental state $\chi^{(n)}$ of Eq. (8) admits a decomposition in terms of translationally invariant matrix product states 20] with finite bound dimensions. Equation (8) also shows that the weakly complementary channel 21], mapping the initial system state to the final environmental one, is given by $\tilde{\Lambda}^{(n)}(\rho)=\operatorname{Tr}_{X}\left[U^{(n)}\left(\rho \otimes \chi^{(n)}\right)\left[U^{(n)}\right]^{\dagger}\right]$. By taking $\chi^{(n)}$ in Bell diagonal form it is easy to show that one has $\tilde{\Lambda}^{(n)}(\rho)=\chi_{B D}^{(n)}$ for all $\rho$. Therefore, the CQ channel $\Lambda^{(n)}$ in (8) is always weak-degradable 21], i.e. $\mathcal{T} \circ \Lambda^{(n)}=\tilde{\Lambda}^{(n)}$ with $\mathcal{T}=\tilde{\Lambda}^{(n)}$.

Examples:- We now analyze some examples of teleportation-induced CQ channels.

First, we consider the situation in which Alice and Bob share $n$ perfect Bell pairs $\left|\Psi_{0}\right\rangle^{\otimes n}$ but they unfortunately loose the classical information about the order of the particles (or equivalently a sequence of random swaps has been applied to the qubits controlled by Alice). In this case the shared state between Alice and Bob, $\chi^{(n)}$, becomes a $2 n$-qubit mixture of all possible pairings. As found in 22], the one-way distillable entanglement of $\chi^{(n)}$ is analytically calculated as $D_{1}\left(\chi^{(n)}\right)=\sum_{j=0}^{n / 2} \frac{(2 j+1)^{2}}{2^{n}(n+1)}\left(\begin{array}{c}n+1 \\ n / 2-j\end{array}\right) \log (2 j+1)$ (here $n$ is assumed to be even). Replacing into Eq. (4) and exploiting the fact that $D_{1}$ is exponentially decreasing in $n$ it is easy to find that, as expected, the quantum capacity of the associated process $\mathcal{L}$ is vanishing, i.e. $Q=0$.

As a second example, we consider the case in which the teleportation mediator $\chi^{(n)}$ is obtained by applying (on Alice side) a sequence of unitary local transformations to $n$ Bell states $\left|\Psi_{0}\right\rangle$ which, as in the previous example, are initially shared among Alice and Bob. Specifically we assume that a quantum phase gate with a conditional phase shift $\theta$ is applied on Alice's $1^{\text {st }}$ and $2^{\text {nd }}$ qubits, later the same gate is applied on her $2^{\text {nd }}$ and $3^{\text {rd }}$ one, and so on and so forth for all $n$ qubits. One can easily verify that the resulting state is a translationally invariant matrix product state [20] which is maximally correlated (i.e. it has vanishing projections on the vectors $\left|\Psi_{1,2}\right\rangle$ ). Therefore in this case the bound (5) is tight. In particular, following [7], one can build an optimal code by partition-

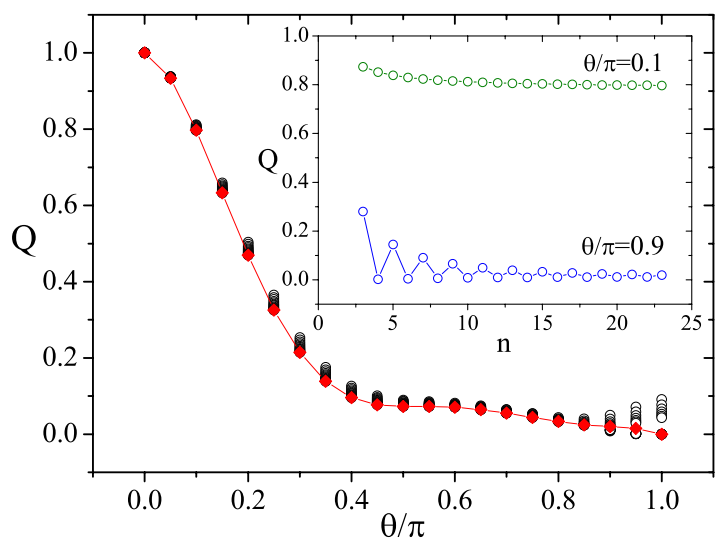

FIG. 2: Quantum capacity $Q(\mathcal{L})$ of the family $\mathcal{L}$ of $\mathrm{CQ}$ channels as a function of the phase shift $\theta / \pi$ for the quantum phase gate example (red diamond). For each value of $\theta / \pi$, the values of $Q^{(n)}$, i.e. $1-S\left(\chi_{B D}^{(n)}\right) / n$, are shown for $n=10, \ldots, 23$ from top to bottom (empty circles). The ideal teleportation is recovered for $\theta=0$, while $Q=0$ for $\theta / \pi=1$ because $\chi^{(n)}$ becomes separable. In the inset, the fast convergence $\left(\lim _{n \rightarrow \infty}\right)$ of $Q^{(n)}$ vs. $n$ is reported for $\theta / \pi=0.1,0.9$. Similar behaviors are found for all values $\theta / \pi \in[0,1]$.

ing the channel's uses in blocks of live and spacer qubits (in our case it is sufficient to have spacer blocks of only two elements each). In Fig. 2 we report the quantum capacity of this channel as a function of the phase shift $\theta / \pi$ computed as in the rhs of Eq. (5). For $\theta=0$ one recovers the ideal teleportation with maximum quantum capacity, while for $\theta=\pi$ the state $\chi^{(n)}$ becomes separable and $Q$ is exactly null.

$C V$ systems:- We now generalize the teleportation representation of $\mathrm{CQ}$ channels to Continuous-Variable (CV) systems. Here we assume that Alice and Bob share a bipartite $2 n$-mode Bosonic state $\chi^{(n)}$ defined on an infinite Hilbert space $\mathcal{H}_{A} \otimes \mathcal{H}_{B}$ which is organized in $n$ couples that are operated independently during a $\mathrm{CV}$ teleportation stage. By following the standard protocol 23], Alice performs the simultaneous quantum measurement of position and momentum for the input system in an arbitrary $n$-mode Bosonic state $\rho$ (to be teleported to Bob) and her part of the bipartite system $\chi^{(n)}$. This is described by a projection on the simultaneous eigenstate of position-difference and momentum-sum. Then Alice informs Bob of the measurement outcome and Bob applies a proper unitary transformation $\hat{V}(z)$ on his part of $\chi^{(n)}$, with $\hat{V}(z)$ being the multi-mode Weyl (displacement) operator (i.e., $\hat{V}(z):=\exp [i \hat{R} z]$ with $z:=$ $\left(x_{1}, x_{2}, \cdots, x_{n}, y_{1}, y_{2}, \cdots, y_{n}\right)^{T}$ being a column vector of $\mathbb{R}^{2 n}$ and $\hat{R}:=\left(\hat{Q}_{1}, \cdots, \hat{Q}_{n} ; \hat{P}_{1}, \cdots, \hat{P}_{n}\right)$, where $\left\{Q_{i}, P_{i}\right\}$ are the canonical coordinates of $n$ modes $[24]$ ). Building up from the results of Ref. [10 for the $n=1$ case, the resulting channel can now be described as the following 
generalized $n$-mode additive classical noise map [25]

$$
\Phi^{(n)}(\rho)=\int d z f^{(n)}(z) \hat{V}(z) \rho \hat{V}(z)^{\dagger}
$$

where $f^{(n)}(z)$ is the probability distribution

$$
f^{(n)}(z)=\operatorname{Tr}\left[E(z) \chi^{(n)}\right]
$$

with $E(z)=\left[\mathbb{1} \otimes \hat{V}(z)^{\dagger}\right] E_{0}^{\otimes n}[\mathbb{1} \otimes \hat{V}(z)]$, and $E_{0}$ being the (not normalized) $\mathrm{CV}$ version of the Bell state [10], i.e. the two-mode infinitely-squeezed vacuum state. Equation (10) generalizes Eq. (2) to the CV case. Here the CJ state becomes

$$
\chi_{C J}^{(n)}=\int d z f^{(n)}(z) E(z)=\int d z E(z) \chi^{(n)} E(z),
$$

which again can be used to replace $\chi^{(n)}$ in the teleportation representation of $\Phi^{(n)}$. Following arguments similar to those used in the qubit case, the quantum capacity $Q(\mathcal{F})$ of a family $\mathcal{F}:=\left\{\Phi^{(n)}: n \in \mathbb{N}\right\}$ can be bounded as in Eqs. (4), and again the inequality saturates for forgetful channels. In the $\mathrm{CV}$ case an upper bound for the distillable entanglement $D_{1}\left(\chi^{(n)}\right)$ is given by the logarithmic negativity $\mathcal{N}$, that is easily computable in terms of the symplectic eigenvalues of the Covariance Matrix $(\mathrm{CM})$ of the CJ state [26]. In particular, let $\chi^{(n)}$ be a $2 n$-mode Gaussian state and $E_{0}$ be the density operator of a two-mode $(r \rightarrow \infty)$-squeezed vacuum state and $\gamma_{E_{0}}$ its CM, i.e. $\gamma_{E_{0}}:=\left[\begin{array}{cc}\Gamma_{-} & 0 \\ 0 & \Gamma_{+}\end{array}\right]$, where $\Gamma_{ \pm}:=\left[\begin{array}{cc}a & b_{ \pm} \\ b_{ \pm} & a_{a}\end{array}\right]$, $a=\nu \cosh (2 r), b_{ \pm}= \pm \nu \sinh (r), \nu$ is the average photon number of the thermal state, and $r$ is the squeezing parameter, which tends to infinity to have a $\mathrm{CV}$ Bell state. Hence, since $E_{0}^{\otimes n}$ is $\gamma_{E_{0}}^{\oplus n}$, we find

$$
f^{(n)}(z)=\frac{2^{2 n} \exp \left[-1 / 2 m^{T}\left(\gamma_{E_{0}}^{\oplus n}+\gamma\right)^{-1} m\right]}{\left[\operatorname{Det}\left(\gamma_{E_{0}}^{\oplus n}+\gamma\right)\right]^{1 / 2}},
$$

with $\gamma$ being the CM of $\chi^{(n)}$ (assumed for the sake of simplicity to have zero mean), and $m:=(0, z)$ being a real vector of dimension $4 n$. Then the logarithmic negativity $\mathcal{N}$ is given by $\mathcal{N}=\left\{\begin{array}{ll}-\sum_{k} \log \tilde{\nu}_{k} & \begin{array}{l}\text { for } k: \tilde{\nu}_{k}<1 \\ \text { otherwise }\end{array} \\ 0\end{array}\right.$, with $\tilde{\nu}_{k}$ the symplectic eigenvalues of the partial transpose of the CM of $\chi_{C J}^{(n)}[26]$.

Conclusions.- We have presented a teleportation representation of correlated Pauli channels and their $\mathrm{CV}$ generalizations which establishes a formal connection between CQ maps and the physics of many-body systems. Consequently, as in Refs. [6, 7, 8], the CQ maps can now be studied in terms of the properties of the multiqubit state $\chi^{(n)}$ which mediates the teleportation. In particular, in the qubit case, by expanding the operators $E_{k}$ as a linear combination of the Pauli operators (e.g., $E_{0}=\sum_{k=0}^{3} \sigma_{k} \otimes \sigma_{k}-2 \sigma_{2} \otimes \sigma_{2}$ ) the probabilities $p_{\mathbf{k}}$ that characterize the channel (10) can be expressed in terms of the $2 n$-point correlation functions of the form
$\operatorname{Tr}\left[\sigma_{\mathbf{k}^{\prime}} \otimes \sigma_{\mathbf{k}^{\prime \prime}} \chi^{(n)}\right]$ (assuming $\chi^{(n)}$ to be the ground state of a many-body Hamiltonian $H$ the latter can then be used to relate the correlations of $\Lambda_{\chi}^{(n)}$ to the physical system couplings).

F.C. and V.G. acknowledge the Quantum Information research program of the Centro E. De Giorgi of SNS for financial support. F.C. was supported also by the EU STREP project CORNER and he would like to thank M.B. Plenio and S. Virmani for discussions.

[1] C. Macchiavello and G. M. Palma, Phys. Rev. A 65 050301(R) (2002); C Macchiavello, G. M. Palma, and S. Virmani, Phys. Rev. A $69010303(\mathrm{R})$ (2004).

[2] G. Bowen and S. Mancini, Phys. Rev. A 69012306 (2004).

[3] D. Kretschmann and R. F. Werner, New J. Phys. 6 26, (2004).

[4] V. Giovannetti, J. Phys. A 38, 10989 (2005).

[5] K. Banaszek et al., Phys. Rev. Lett. 92, 257901 (2004).

[6] V. Giovannetti and S. Mancini, Phys. Rev. A 71, 062304 (2005).

[7] M. B. Plenio and S. Virmani, Phys. Rev. Lett. 99, 120504 (2007); New J. Phys. 10, 043032 (2008).

[8] D. Rossini, V. Giovannetti, and S. Montangero, New. J. Phys. 10115009 (2008) .

[9] G. Bowen and S. Bose, Phys. Rev. Lett. 87, 267901 (2001)

[10] M. Ban, M. Sasaki, and M. Takeoka, J. Phys. A: Math. Gen. 35, L401 (2002).

[11] C. H. Bennett, et al., Phys. Rev. Lett. 70, 1895 (1993).

[12] A. Jamiolkowski, Rep. Math. Phys. 3, 275 (1972); M.-D. Choi, Linear Algebr. Appl. 10, 285 (1975).

[13] W. Roga, et al. J. Phys. A: Math. Theor. 41, 035305 (2008).

[14] M. A. Nielsen and I. L. Chuang, Quantum Computation and Quantum Information (CUP, Cambridge 2000).

[15] C. H. Bennett, et al., Phys. Rev. A 54, 3824 (1996).

[16] I. Devetak, IEEE Trans. Inf. Theory 51, 44 (2005).

[17] I. Devetak and A. Winter, Proc. R. Soc. Lond. A 461, 207 (2005).

[18] E. M. Rains, Phys. Rev A 60, 173 (1999); ibid. 60, 179 (1999); IEEE Trans. Inf. Th. 47, 2921 (2001).

[19] A. D'Arrigo, G. Benenti, and G. Falci, New J. Phys. 9, 310 (2007).

[20] M. Fannes, B. Nachtergaele, and R. F. Werner, Commun. Math. Phys. 144443 (1992).

[21] F. Caruso and V. Giovannetti, Phys. Rev. A 74, 062307 (2006); F. Caruso, V. Giovannetti, and A.S. Holevo, New J. Phys. 8, 310 (2006).

[22] J. Eisert, et al., Phys. Rev. Lett. 84, 1611 (2000).

[23] S. L. Braunstein, and H. J. Kimble, Phys. Rev. Lett. 80, 869 (1998); A. Furusawa et al., Science 282706 (1998).

[24] A. S. Holevo, Probabilistic and Statistical Aspects of Quantum Theory (Amsterdam: North-Holland, 1982).

[25] A. S. Holevo and R. F. Werner, Phys. Rev. A 63, 032312 (2001).

[26] G. Vidal and R. F. Werner, Phys. Rev. A 65, 032314 (2002). 